\title{
Effects of the Great East Japan Earthquake on Secondary Sex Ratio and Perinatal Outcomes
}

\author{
Kohta Suzuki ${ }^{1}$, Zentaro Yamagata ${ }^{1}$, Miyuki Kawado ${ }^{2}$, and Shuji Hashimoto ${ }^{2}$ \\ ${ }^{1}$ Department of Health Sciences, Interdisciplinary Graduate School of Medicine and Engineering, University of Yamanashi, Chuo, Yamanashi, Japan \\ ${ }^{2}$ Department of Hygiene, Fujita Health University School of Medicine, Toyoake, Aichi, Japan
}

Received March 8, 2015; accepted June 20, 2015; released online December 5, 2015

Copyright $(\odot 2015$ Kohta Suzuki et al. This is an open access article distributed under the terms of Creative Commons Attribution License, which permits unrestricted use, distribution, and reproduction in any medium, provided the original author and source are credited.

\begin{abstract}
Background: The effect of natural disasters on secondary sex ratio (SSR) and perinatal outcomes has been suggested. This study aimed to examine effects of the Great East Japan Earthquake on perinatal outcomes using vital statistics of Japan.

Methods: Birth registration data from vital statistics of Japan between March 2010 and March 2012 were used. Pregnant women who experienced the earthquake were categorized according to their gestational period as of March 11, 2011, as follows: gestational weeks 4-11, 12-19, 20-27, and 28-36 (2011 group). Similarly, pregnant women who did not experience the earthquake were categorized according to their gestational period as of March 11, 2010 and used as controls (2010 group). We also categorized prefectures as "extremely affected", "moderately affected", and "slightly or unaffected" regions. SSR, birth weight, and gestational period were compared between both groups. Results: The number of singleton births was 688479 in the 2010 group and 679131 in the 2011 group. In the extremely affected region, the SSR among women at 4-11 weeks of gestation was significantly lower in the 2011 group compared with the 2010 group $(49.8 \%$ vs $52.1 \%, P=0.009)$. In the extremely affected region, children born to women who experienced the earthquake at 28-36 weeks of gestation had significantly lower birth weights.

Conclusions: The SSR declined among women who experienced the earthquake during early pregnancy, particularly in the extremely affected region. However, no apparent negative effect of the earthquake on perinatal outcomes was observed, although birth weight of infants who were born to women who experienced the earthquake at $28-36$ weeks of gestation were lower.
\end{abstract}

Key words: maternal stress; natural disaster; secondary sex ratio; gestational duration; birth weight

\section{INTRODUCTION}

The effect of natural disasters, such as earthquakes, on perinatal outcomes has been previously examined. For instance, in Chile, expectant mothers who experienced the strong earthquake in 2005 were found to be likely to deliver babies before full term, especially for female children. As a result, the ratio of males to females at birth (secondary sex ratio $[\mathrm{SSR}])$ was decreased among these women. ${ }^{1}$ Further, in another report that examined the effects of the earthquake in Chile in 2010, Oyarzo et al reported that pregnant women who experienced the earthquake during early pregnancy were more likely to deliver low-birth-weight (LBW) infants than pregnant women who experienced the earthquake during late pregnancy. ${ }^{2}$ In contrast, a systematic review by Harville et al that examined the associations between natural disasters and perinatal outcomes suggested that women who experienced these disasters were likely to deliver small-for-gestational-age (SGA) infants, ${ }^{3}$ with a relatively small effect on gestational age. In Japan, Fukuda et al examined SSR after the Kobe earthquake in 1995; they concluded that at 9 months after the earthquake, the SSR was significantly lower than prior to the earthquake. ${ }^{4}$

It appears to be widely accepted that the SSR decreases following natural disasters. However, this effect is not fully understood. In addition, these previous studies had certain limitations. For example, although an effect of seasonality has been noted for $\mathrm{SSR}^{5-7}$ or preterm births, ${ }^{8}$ none of the above studies strictly adjusted for seasonal changes in perinatal outcomes. Further, studies using individual data for analysis 
had relatively small sample sizes, while studies with larger numbers of subjects were designed as ecological studies. Thus, it is difficult to determine the causal association between natural disasters and perinatal outcomes.

On March 11, 2011, a huge earthquake occurred in eastern Japan, called the Great East Japan Earthquake, inducing a subsequent massive tsunami. Millions of people were affected by this earthquake and tsunami, which was determined to be the most severe natural disaster in Japan in recorded history. ${ }^{9}$ By March 11, 2014, casualties included 15884 deaths, 2633 missing, and 6148 injured, as confirmed by the National Police Agency of Japan. ${ }^{10}$ The most severely affected prefectures were Iwate, Miyagi, and Fukushima. Catalano et al examined the effect of this earthquake on SSR using birth registration data from the vital statistics data of Japan and suggested that sensitive periods during both early and late pregnancy led to a decreased SSR. ${ }^{11}$ Further, in the most severely affected prefectures, the rate of conception of males was reduced. ${ }^{11}$ However, these results were based on aggregate data, and these effects have not yet been examined on an individual level.

This study aimed to examine the effects of the Great East Japan Earthquake on SSR, birth weight, and gestational duration in weeks using individual birth registration data in the most severely affected prefectures and other prefectures in Japan.

\section{METHODS}

\section{Birth registration data}

In Japan, every birth must be registered by law. Thus, the study participants consisted of all births that occurred during the study period. The birth registration data are filed at relevant city health departments and entered into computerized files at the Ministry of Health, Labour and Welfare of Japan. Individual birth registration data from these vital statistics of Japan between March 2010 and March 2012 were used for this study. These data were anonymously provided under the Statistics Act in Japan and contain the birthplace, birth date, child's sex, birth weight, gestational age, parity, and ages of the father and mother. Except for the ages of the parents, the obstetrician who attended the birth provided this information on the birth certificate. In this study, it was not necessary for participants to provide informed consent because every birth in Japan is legally required to be registered, and birth registration data are available for researchers to use with permission from the Ministry of Health, Labour and Welfare under the Statistics Act in Japan.

To examine the effect of the earthquake on SSR and perinatal outcomes, we compared two groups of pregnant women. Pregnant women who experienced the earthquake at 4-36 weeks of gestation were categorized according to their gestational period as of March 11, 2011, as follows: gestational weeks 4-11, 12-19, 20-27, and 28-36 (2011 group, $n=679131$ ). Pregnant women who did not experience the earthquake were similarly categorized according to their gestational period as of March 11, 2010, and used as controls (2010 group, $n=688479$ ). The gestational duration at the time of the earthquake was calculated by using the birth date and gestational age from the birth registration data. Because an effect of the earthquake on preterm birth and a gender difference in this effect have been suggested, ${ }^{1}$ women who experienced the earthquake after 37 weeks of gestation were excluded to examine the effect of earthquake on SSR and preterm birth. In addition, stillbirths were also excluded because the purpose of this study was only to examine the effect of the earthquake on live births. We also categorized prefectures as "extremely affected" (Iwate, Miyagi, and Fukushima; more than 100 dead or missing), "moderately affected" (more than 10 injured and less than or equal to 100 dead or missing), and "slightly or unaffected" (Figure).

Because this study was conducted using anonymous data, which was provided under the Statistics Act in Japan, no ethical consideration was needed.

\section{Statistical analysis}

To examine the effect of the earthquake on SSR and perinatal outcomes, we compared the above-mentioned 2011 and 2010 groups. Analyses were also conducted for the "extremely affected", "moderately affected", and "slightly or unaffected" regions. Further, only singleton babies were analysed because of known significant differences in gestational duration and birth weight between singleton and multiple births. ${ }^{12}$ First, the mean gestational duration, mean birth weight, mean maternal age at birth, mean birth order, prevalence of preterm birth, prevalence of LBW infants, and rate of first-born births were compared between groups in each region by sex. Student's $t$-tests were used as crude analyses for mean gestational duration and mean birth weight. Chi-square tests were performed as crude analyses for categorical variables. Next, the SSRs of all births were compared between the 2010 and 2011 groups in each gestational period and region. Chi-square tests were conducted to analyse the differences. Finally, in each gestational period and region, multiple linear regression analyses were conducted to examine the effect of the earthquake on gestational duration after controlling for confounding factors, such as maternal age and parity. Similar analyses for birth weight were conducted to adjust for maternal age, parity, and gestational duration. In addition, based on the results of multiple regression analyses, adjusted mean gestational duration and birth weight were calculated by the least-squares method. These analyses were conducted in each gender because gender differences in effects of the earthquake have been suggested. ${ }^{1}$ All analyses were conducted using SAS version 9.3 (SAS Institute, Inc., Cary, NC, USA). 


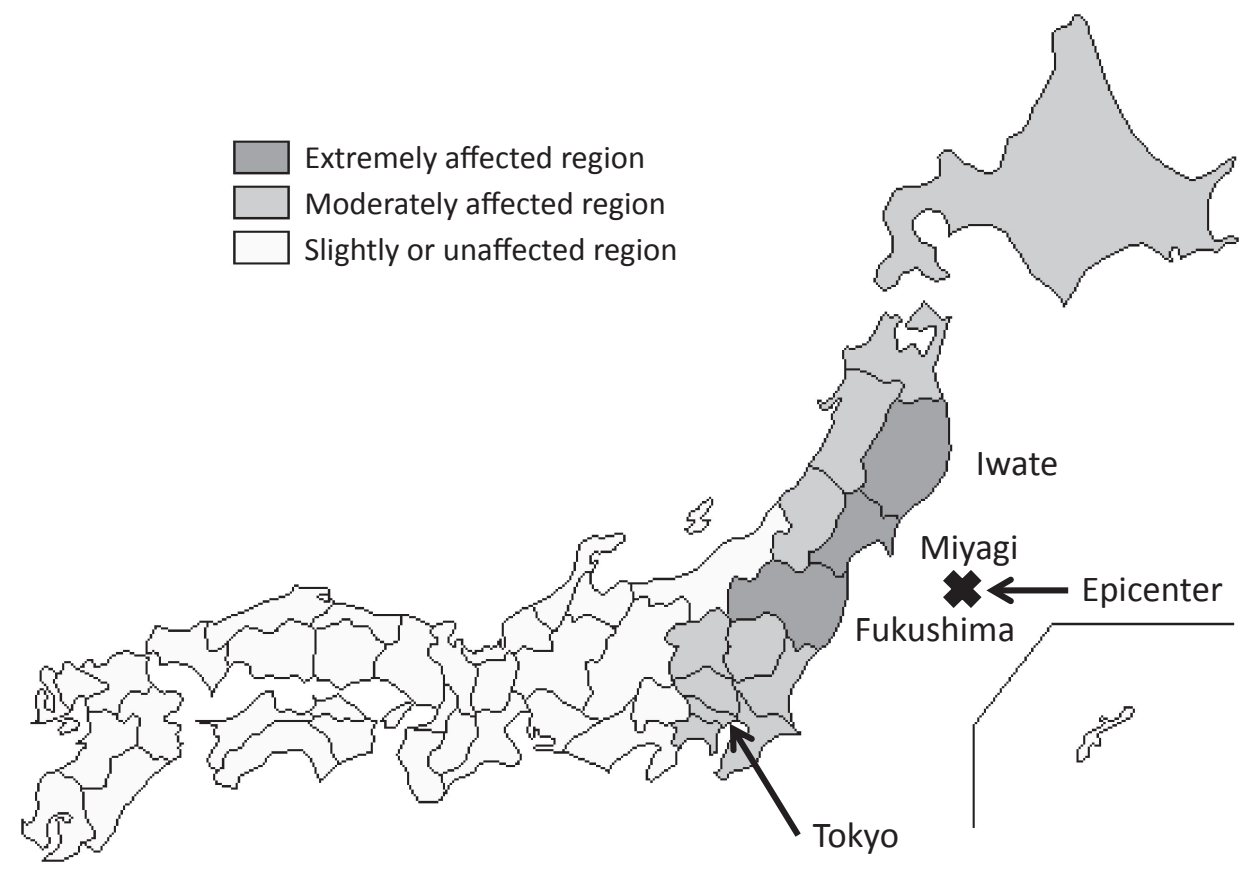

Figure. Categorization of prefectures by the extent of damage caused by the earthquake.

\section{RESULTS}

\section{Study population}

The characteristics of the study population are described in Table 1. The numbers of singleton babies born in 2010 and 2011 were 676124 (348040 boys and 328084 girls) and 667139 (342344 boys and 324795 girls), respectively. The mean maternal age at birth in the 2011 group was significantly higher than in the 2010 group in almost every region. Moreover, in the 2011 group, the mean birth order was significantly greater and the rate of first-born babies was lower than in the 2010 group. However, no significant differences in gestational duration, birth weight, prevalence of preterm birth, or rate of LBW infants were observed in the extremely affected region between the 2010 and 2011 groups.

\section{Analysis of the SSRs}

In the extremely affected region, the male-to-female ratio among the women at 4-11 weeks of gestation was significantly lower in the 2011 group than in the 2010 group (49.8\% in the 2011 group and $52.1 \%$ in the 2010 group; $P=0.009$ ) (Table 2). However, the 2010 and 2011 groups did not significantly differ during other gestational periods. Further, in the moderately affected region, there was no significant difference between the 2010 and 2011 groups in each gestational period. In contrast, although the difference in SSR was relatively small, a significant decrease in SSR was noted between the 2010 and 2011 groups (51.7\% vs 50.9\%) among the women in the slightly or unaffected region at 4-11 weeks of gestation $(P=0.0011)$. However, there were no significant differences between the 2010 and 2011 groups during other gestational periods in this region.

\section{Linear regression models for gestational duration and birth weight}

No significant decline in gestational duration was observed in the 2011 group compared with the 2010 group for either sex (Table 3). However, in the extremely affected region, a significant decrease in birth weight was observed for male children of women who had been at 28-36 weeks of gestation during the earthquake compared with the 2010 group. However, there was no significant decrease in birth weight in the 2011 group compared with the 2010 group among women in other gestational periods. Further, in the moderately affected and the slightly or unaffected region, no significant decline in the gestational duration or birth weight was noted in the 2011 group compared with the 2010 group for either sex.

\section{DISCUSSION}

This study is the first to examine the effects of the Great East Japan Earthquake on SSR and perinatal outcomes, with consideration of the seasonal patterns of perinatal indicators. Our results suggested that SSR declined in women who experienced the earthquake during their early pregnancy, particularly for those in the extremely affected region. For perinatal outcomes, such as gestational duration and birth weight, no significant negative effects of the earthquake were observed in the moderately affected or slightly affected regions; however, in the extremely affected region, adjusted mean birth weight was smaller for male children delivered by women who experienced the earthquake at 28-36 weeks of gestation.

Some demographic differences were noted between the 2010 and 2011 groups. However, the total fertility rates of 


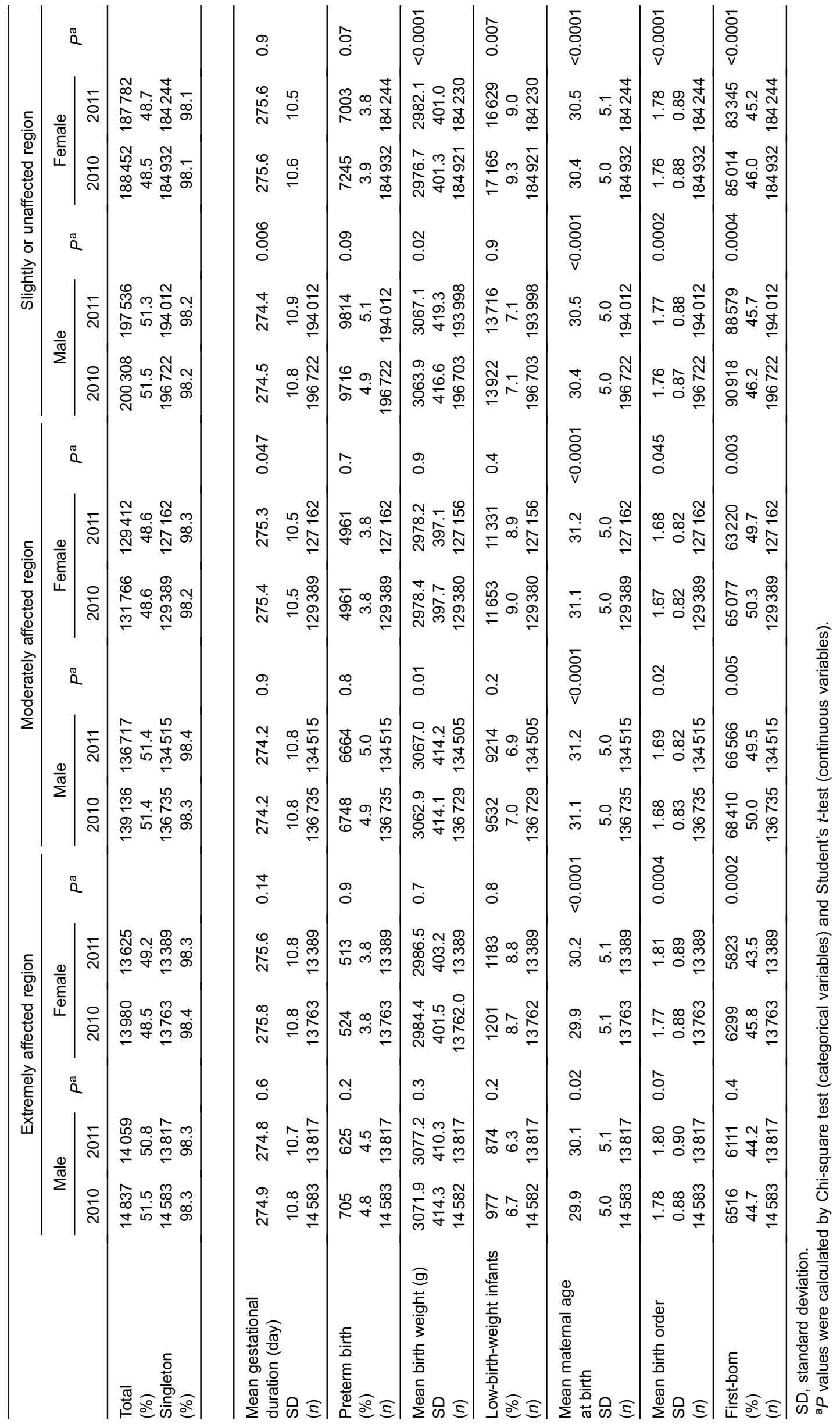


Table 2. Comparison of secondary sex ratio (SSR) of all singleton babies between the 2010 and 2011 groups in each gestational period category and region

\begin{tabular}{|c|c|c|c|c|c|c|c|c|c|c|c|c|}
\hline \multirow{2}{*}{ Gestational weeks } & \multicolumn{3}{|c|}{ 4-11 weeks } & \multicolumn{3}{|c|}{$12-19$ weeks } & \multicolumn{3}{|c|}{$20-27$ weeks } & \multicolumn{3}{|c|}{ 28-36 weeks } \\
\hline & Total & Male & $p^{a}$ & Total & Male & $P$ & Total & Male & $P$ & Total & Male & $P$ \\
\hline \multicolumn{13}{|l|}{ Extremely affected region } \\
\hline 2010 group & 6798 & 3541 & 0.009 & 7082 & 3680 & 0.66 & 6948 & 3499 & 0.07 & 7518 & 3863 & 0.07 \\
\hline SSR & & 52.1 & & & 52.0 & & & 50.4 & & & 51.4 & \\
\hline 2011 group & 6618 & 3298 & & 6716 & 3465 & & 6649 & 3450 & & 7223 & 3604 & \\
\hline SSR & & 49.8 & & & 51.6 & & & 51.9 & & & 49.9 & \\
\hline \multicolumn{13}{|c|}{ Moderately affected region } \\
\hline 2010 group & 66757 & 34155 & 0.97 & 65525 & 33949 & 0.86 & 65018 & 33251 & 0.28 & 68824 & 35380 & 0.61 \\
\hline SSR & & 51.2 & & & 51.8 & & & 51.1 & & & 51.4 & \\
\hline 2011 group & 65137 & 33320 & & 64947 & 33617 & & 63721 & 32780 & & 67872 & 34798 & \\
\hline SSR & & 51.2 & & & 51.8 & & & 51.4 & & & 51.3 & \\
\hline \multicolumn{13}{|c|}{ Slightly or unaffected region } \\
\hline 2010 group & 94792 & 48980 & 0.0011 & 94700 & 48466 & 0.43 & 92986 & 48127 & 0.21 & 99176 & 51149 & 0.48 \\
\hline SSR & & 51.7 & & & 51.2 & & & 51.8 & & & 51.6 & \\
\hline 2011 group & 93363 & 47541 & & 94709 & 48641 & & 91825 & 47257 & & 98359 & 50573 & \\
\hline SSR & & 50.9 & & & 51.4 & & & 51.5 & & & 51.4 & \\
\hline
\end{tabular}

${ }^{\text {a }} P$ values were calculated by Chi-square test.

women during these years were similar. ${ }^{13}$ In addition, these differences were very small, although some were significant because of the huge sample size. In fact, there appeared to be few significant effects of the earthquake on perinatal outcomes. Thus, these differences, particularly the difference in SSR, might not be associated with the earthquake. Moreover, although SSR in the 2011 group also significantly decreased in the slightly or unaffected regions compared with the 2010 group, the effect of the earthquake on SSR may have been limited in the extremely affected region. The difference in SSR between the slightly and unaffected region $(51.7 \%$ in the 2010 group vs $50.9 \%$ in the 2011 group among women at 4-11 weeks of gestation) was statistically significant; however, no such significant difference was observed in the extremely affected region. In the extremely affected region, there was no significant difference in SSR among women who experienced the earthquake at 20-27 and 28-36 weeks of gestation, although these differences were larger than the differences among the women at 4-11 weeks of gestation in the slightly or unaffected region. We believe that the statistically significant difference seen in the slightly or unaffected region could merely be a result of the large study population. Therefore, it is difficult to assess whether or not this difference was a result of the earthquake.

With respect to SSR, our results were largely consistent with those of previous studies. Catalano et al examined the effects of the same earthquake and concluded that rate of male births decreased because of decreased conception rates of male foetuses and increased selection of female foetuses in utero. ${ }^{11}$ However, they only examined the number of births in each month, since they analysed aggregate birth registration data. Thus, their results did not consider the stressors of the earthquake at the individual level. Despite differing methodologies, our results concerning the effects of the earthquake during early pregnancy were consistent with their findings. Further, our results, which suggested that experiencing the earthquake during early pregnancy was associated with decreased SSR, were largely consistent with the results of Fukuda et al, although they also analysed aggregated birth registration data concerning the Kobe earthquake. ${ }^{4}$ However, our findings regarding the effect of the earthquake during late pregnancy differed somewhat from those of Catalano et al. ${ }^{11}$ Where Catalano et al suggested that there might be a sensitive period in late gestation, our results suggested that SSR was not significantly different and that the birth weight in males was likely to be lower when the mothers experienced the earthquake at 28-36 weeks of gestation. However, in our analysis, it was impossible to assess the effect of the earthquake after 37 weeks of gestation because we excluded women who experienced the earthquake after that point from the analysis. Therefore, in women who experienced the earthquake after 37 weeks of gestation, it was suggested by the previous reports that the gestational duration for male children was likely to be shortened because of intrauterine growth restriction, ${ }^{14}$ with a resultant decline in SSR during late pregnancy. However, because the adjusted difference in mean birth weight between 2010 and 2011 was only $16 \mathrm{~g}$, the effect of the earthquake on foetal growth might be small.

The present findings were also largely consistent with results of a study by Torche and Kleinhaus concerning the impact on SSR of the 2005 earthquake in Chile, which analysed individual birth registration data and concluded that the SSR decreased among women who experienced that earthquake. ${ }^{1}$ They also demonstrated that expectant mothers who experienced that earthquake were likely to deliver babies before full term, especially for female children, ${ }^{1}$ although we noted no significant effect of the earthquake on shortened 


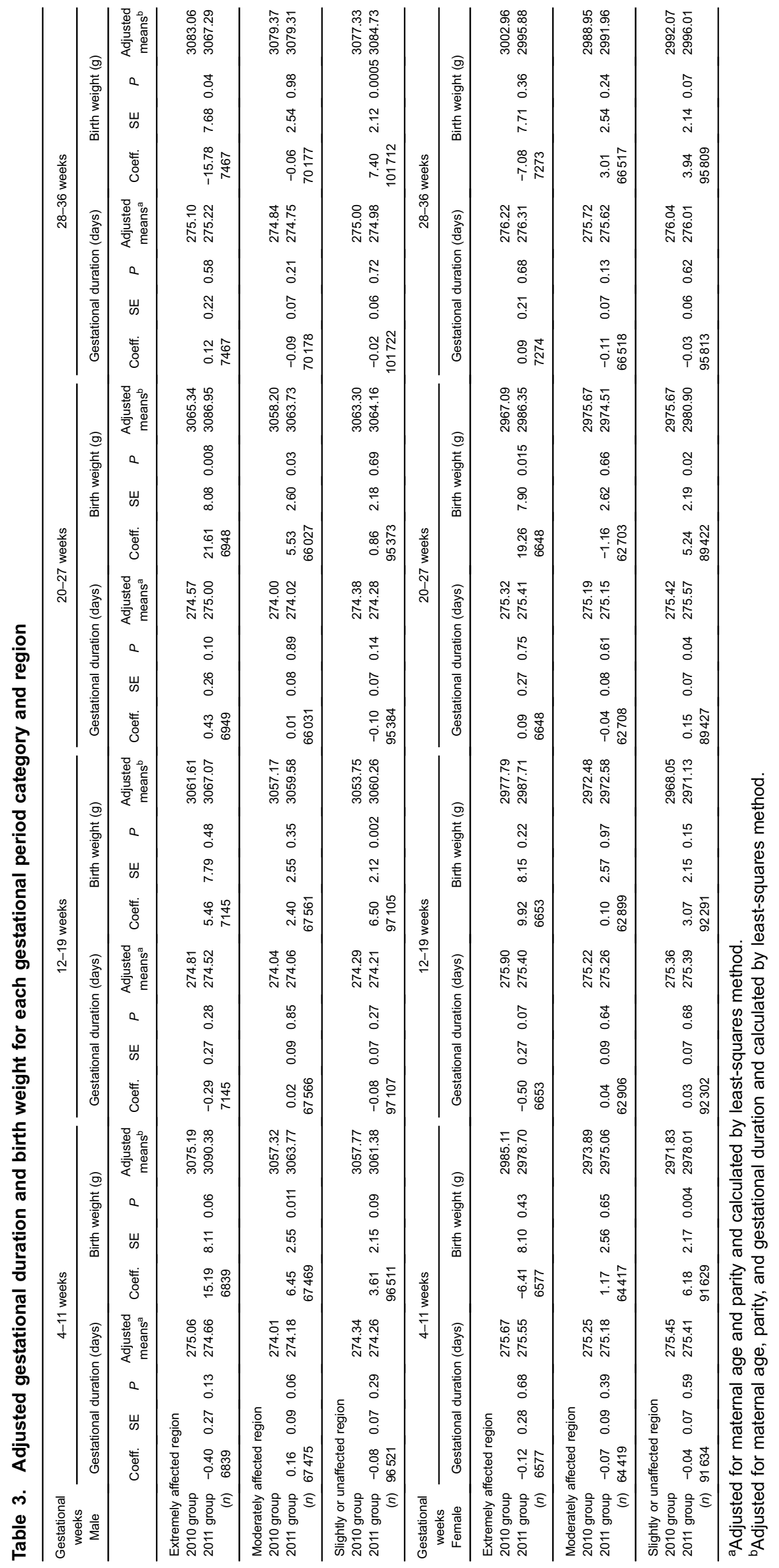


gestational duration. However, only in Iwate Prefecture, which is part of the extremely affected region, a significant decrease in gestational duration for female children was observed among women who experienced the earthquake during early pregnancy (data not shown). Therefore, our results in this prefecture were similar to their results although the number of population in this prefecture was limited.

It has been suggested that cohorts that have experienced severe maternal stress during pregnancy, such as that caused by natural disasters, have lower-than-expected SSRs. ${ }^{15,16}$ Natural selection is hypothesized to include conservation mechanisms by which stressed pregnant females preferentially cull frail male foetuses. This hypothesis, known as the TriversWillard hypothesis, ${ }^{17}$ proposes that adverse maternal health shocks around conception or during pregnancy lead to a sex ratio skewed in favour of female offspring, and the present results support this hypothesis. One possible explanation for the hypothesis is that, because male foetuses grow faster and larger than female foetuses, ${ }^{18}$ male foetuses may require more resources from the mother and may be less likely to adapt their development to a stressful intrauterine environment. ${ }^{1}$ On the other hand, female foetuses might need fewer resources than males for development in utero ${ }^{1}$ and may show reduced growth and demands on the mother in response to maternal stress. ${ }^{19}$ Finally, male newborns are also more likely to die than females. ${ }^{20}$

Previous studies regarding the effects of earthquakes on perinatal outcomes, such as the report regarding the Chile earthquake, have indicated that expectant mothers who experience the earthquake during early pregnancy are likely to have preterm deliveries. ${ }^{2}$ In our analyses, some negative effects of the earthquake on gestational duration and birth weight were only observed in part of the extremely affected region. Moreover, no clear effect of the earthquake on perinatal outcomes was observed. This discrepancy between the results of our study and those of the study conducted in Chile might be caused by differences in geographical factors and medical support systems after natural disasters in each country.

This study has several limitations that warrant mention. First, to control for the seasonality of SSR and gestational duration, women who were pregnant at 1 year before the earthquake were used as controls. It is possible that this interval was not adequate to control for seasonality; however, very few studies have previously considered the seasonality of SSR and perinatal outcomes. Second, since Japanese birth registration data do not include information regarding maternal lifestyle and complications during pregnancy, it was difficult to adjust for potential confounders of perinatal outcomes, such as maternal smoking during pregnancy; however, we did control for maternal age at birth and birth order. In addition, the method of calculating gestational age was not specified in the vital statistics, although information concerning gestational age was provided by obstetricians. In any case, the method for determining gestational age is likely to have been the same in 2010 and 2011 as well as between each gestational week category of participants and among regions, because there is a guideline for obstetrical practice in Japan. ${ }^{21}$ In addition, this kind of measurement error is non-differential. As a result, this study limitation would be expected to reduce the statistical power of comparison between 2010 and 2011 groups. Thus, it is unlikely that detected differences in SSR were due to chance, as nondifferential errors generally make variance of distribution larger. Particularly in the analyses of perinatal outcomes, there might be certain type I errors due to multiple comparisons because many comparisons were made between the 2010 and 2011 groups in each gestational period and region. However, for the comparison of SSR in the extremely affected region, we expect our results to be robust because the $P$ value was very small. Further, since the earthquake, emigration from the disaster-stricken areas has increased, particularly from Fukushima Prefecture. ${ }^{22}$ In addition, the number of deliveries, including deliveries of women who went to their parents' home and delivered at a hospital in their parents' town to obtain perinatal support from their parents (which is called a "Satogaeri" delivery), decreased in the extremely affected region after the earthquake due to problems of gaining access to medical facilities because of the shutdown of some facilities. ${ }^{23}$ These factors may have resulted in differences between the 2010 and 2011 groups. However, the effect of population movement might be limited because there was no significant difference between the 2010 and 2011 groups in other areas, though some people migrated from the extremely affected region. Further, it was impossible to examine differences in the effects between the coastal areas, which were affected by the tsunami, and other areas. The amount of data we obtained from municipalities in the coastal and mountainous areas was relatively small. Therefore, we could not differentiate between the effects of the earthquake versus those of the tsunami. However, if only the effects of the tsunami existed, the results would be underestimated because the effects of the tsunami was very few in other areas. Finally, we did not examine the effect of the earthquake on rates of abortions and stillbirths, which might be essential to clarify the mechanism of differences in SSR. However, our results support previous hypotheses about the mechanism of such differences that do not rely on abortions and stillbirths, since the present findings were mostly consistent with those of previous studies.

Despite these limitations, we consider our results to be valuable for examining the biological mechanisms underlying the effects of natural disasters on SSR and perinatal outcomes, particularly since this study analysed huge datasets from national individual birth registration records.

In conclusion, this study is the first to analyse the effects of the Great East Japan Earthquake on perinatal outcomes using individual data, with consideration of the seasonal patterns of perinatal indicators. Our findings suggest that SSR declined 
among women who experienced the earthquake during early pregnancy, particularly in the extremely affected region. However, with regard to perinatal outcomes, such as gestational duration and birth weight, no apparent negative effect of the earthquake was observed, although birth weight of infants who were born to women who experienced the earthquake at 28-36 weeks of gestation were lower.

\section{ONLINE ONLY MATERIAL}

Abstract in Japanese.

\section{ACKNOWLEDGEMENTS}

This work was supported by Health Labour Sciences Research Grant (H24-Toukei-Ippan-006) from the Ministry of Health, Labour and Welfare, Japan.

The authors thank the research team, which examined the effects of the Great East Japan Earthquake on health status by statistical data, for critical discussion.

We would like to thank Editage (www.editage.jp) for English language editing.

Conflicts of interest: None declared.

\section{REFERENCES}

1. Torche F, Kleinhaus K. Prenatal stress, gestational age and secondary sex ratio: the sex-specific effects of exposure to a natural disaster in early pregnancy. Hum Reprod. 2012;27: 558-67.

2. Oyarzo C, Bertoglia P, Avendaño R, Bacigalupo F, Escudero A, Acurio J, et al. Adverse perinatal outcomes after the February 27th 2010 Chilean earthquake. J Matern Fetal Neonatal Med. 2012;25:1868-73.

3. Harville E, Xiong X, Buekens P. Disasters and perinatal health: a systematic review. Obstet Gynecol Surv. 2010;65:713-28.

4. Fukuda M, Fukuda K, Shimizu T, Møller H. Decline in sex ratio at birth after Kobe earthquake. Hum Reprod. 1998;13:2321-2.

5. James WH. Interpregnancy intervals, high maternal age and seasonal effects on the human sex ratio. Hum Reprod. 1996;11: 7-8.

6. Lerchl A. Seasonality of sex ratio in Germany. Hum Reprod. 1998;13:1401-2.

7. Melnikov VN, Skosyreva GA, Krivoschekov SG. Seasonality bias in adverse pregnancy outcomes in Siberia. Alaska Med. 2007;49 Suppl 2:218-20.
8. Currie J, Schwandt H. Within-mother analysis of seasonal patterns in health at birth. Proc Natl Acad Sci USA. 2013;110: 12265-70.

9. Geller RJ. Shake-up time for Japanese seismology. Nature. 2011;472:407-9.

10. National Police Agency of Japan. 2014. Police White Paper (in Japanese). Available at: https://www.npa.go.jp/archive/keibi/ biki/higaijokyo.pdf. Accessed 2014 March 14.

11. Catalano R, Yorifuji T, Kawachi I. Natural selection in utero: evidence from the Great East Japan Earthquake. Am J Hum Biol. 2013;25:555-9.

12. Blondel B, Kogan MD, Alexander GR, Dattani N, Kramer MS, Macfarlane A, et al. The impact of the increasing number of multiple births on the rates of preterm birth and low birthweight: an international study. Am J Public Health. 2002;92:1323-30.

13. Statistics and Information Department, Minister's Secretariat, Ministry of Health, Labour and Welfare. Vital Statistics of Japan. Tokyo: Health and Welfare Statistics Association; 2012.

14. Rueness J, Vatten L, Eskild A. The human sex ratio: effects of maternal age. Hum Reprod. 2012;27:283-7.

15. Bruckner TA, Catalano R, Ahern J. Male fetal loss in the U.S. following the terrorist attacks of September 11, 2001. BMC Public Health. 2010;10:273.

16. Catalano R, Zilko CE, Saxton KB, Bruckner T. Selection in utero: a biological response to mass layoffs. Am J Hum Biol. 2010;22:396-400.

17. Trivers RL, Willard DE. Natural selection of parental ability to vary the sex ratio of offspring. Science. 1973;179:90-2.

18. de Zegher F, Devlieger H, Eeckels R. Fetal growth: boys before girls. Horm Res. 1999;51:258-9.

19. Sandman CA, Davis EP, Buss C, Glynn LM. Exposure to prenatal psychobiological stress exerts programming influences on the mother and her fetus. Neuroendocrinology. 2012;95:7-21.

20. Bhaumik U, Aitken I, Kawachi I, Ringer S, Orav J, Lieberman E. Narrowing of sex differences in infant mortality in Massachusetts. J Perinatol. 2004;24:94-9.

21. Minakami H, Hiramatsu Y, Koresawa M, Fujii T, Hamada H, Iitsuka Y, et al. Guidelines for obstetrical practice in Japan: Japan Society of Obstetrics and Gynecology (JSOG) and Japan Association of Obstetricians and Gynecologists (JAOG) 2011 edition. J Obstet Gynaecol Res. 2011;37:1174-97.

22. Higuchi Y, Inui T, Hosoi T, Takabe I, Kawakami A. The impact of the Great East Japan Earthquake on the labor market - need to resolve the employment mismatch in the disaster-stricken areas. Japan Labor Review. 2012;9(4):4-21.

23. Sanuki R, Satoh E, Kumakawa T, Yoshikawa T. Accessibility to medical care in case of major disasters. J Natl Inst Public Health. 2013;62:52-61. 